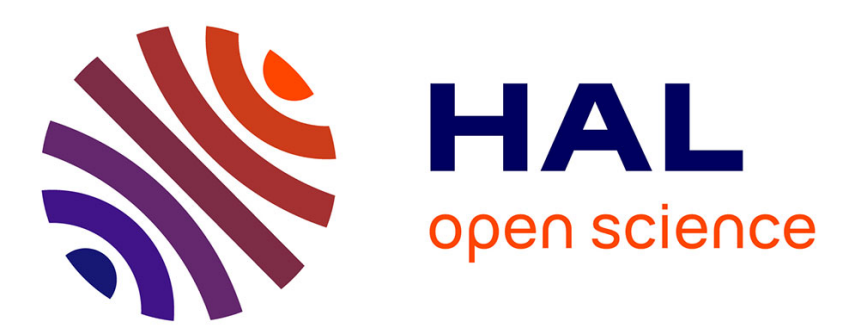

\title{
Spectromètre de paires utilisant des chambres a étincelles à lecture par ligne magnétostrictive
}

P.Y. Bertin, J. Castor, A. Devaux, D.B. Isabelle, M. Lieutier, E.J. Planchard

\section{To cite this version:}

P.Y. Bertin, J. Castor, A. Devaux, D.B. Isabelle, M. Lieutier, et al.. Spectromètre de paires utilisant des chambres a étincelles à lecture par ligne magnétostrictive. Revue de Physique Appliquée, 1973, 8 (1), pp.67-73. 10.1051/rphysap:019730080106700 . jpa-00243655

\section{HAL Id: jpa-00243655 https://hal.science/jpa-00243655}

Submitted on 1 Jan 1973

HAL is a multi-disciplinary open access archive for the deposit and dissemination of scientific research documents, whether they are published or not. The documents may come from teaching and research institutions in France or abroad, or from public or private research centers.
L'archive ouverte pluridisciplinaire HAL, est destinée au dépôt et à la diffusion de documents scientifiques de niveau recherche, publiés ou non, émanant des établissements d'enseignement et de recherche français ou étrangers, des laboratoires publics ou privés. 


\title{
SPECTROMẼTRE DE PAIRES UTILISANT DES CHAMBRES A ÉTINCELLES A LECTURE PAR LIGNE MAGNÉTOSTRICTIVE
}

\author{
P. Y. BERTIN, J. CASTOR, A. DEVAUX, D. B. ISABELle, M. LIEUTIER et E. J. PlanChaRd \\ UER des Sciences Exactes et Naturelles, F 63 Clermont-Ferrand
}

(Reçu le 30 mai 1972, révisé le 18 septembre 1972)

\begin{abstract}
Résumé. - Nous décrivons un spectromètre de paires comportant des chambres à étincelles à lecture par magnétostriction utilisé pour étudier la matérialisation de photons dans le champ des électrons atomiques. Un calculateur Varian $620 \mathrm{i}$ en ligne permet de stocker les données sur bandes magnétiques.
\end{abstract}

Abstract. - A pair spectrometer including spark chambers with magnetostrictive read-out is described. It was used to study photon materialization in the field of atomic electrons. A Varian $620 \mathrm{i}$ computer allows an on-line storing of the data on magnetic tapes.

1. Introduction. - De nombreuses expériences ont permis de déterminer la section efficace totale de matérialisation de photons, produits par rayonnement de freinage, dans le champ de l'électron atomique (triplets) ; cependant, les résultats obtenus ne sont pas en général en accord avec la théorie [1], [2]. Une étude précédente [2] effectuée à l'aide de l'émulsion nucléaire dans notre laboratoire, a montré que la section efficace totale de triplets est en bon accord avec les théories de Borsellino [3] et Mork [1]. Cependant, les difficultés de dépouillement et le nombre importảnt de faux triplets (évalué à $44 \%$ ) rendaient souhaitable une nouvelle expérience dans laquelle un dépouillement rapide et sûr s'accompagnerait d'un nombre négligeable de faux événements. Quelques expériences réalisées notamment par Hart et al. [4], Gates et al. [5], Benaksas et Morrison [6] donnent les distributions de l'impulsion $q$ de l'électron de recul ainsi que l'angle $\theta_{\boldsymbol{r}}$ d'émission de cet électron par rapport à la direction du faisceau de photons ; toutefois, ces distributions, ainsi que la section efficace totale n'ont été mesurées que pour de larges bandes d'énergie de photons et la distribution du recul est imprécise en dessous de $100 \mathrm{keV}$. Enfin, à notre connaissance, l'angle d'ouverture de la paire (ouverture projetée sur un plan) a seulement été mesuré par Hart et al. [4]. Dans le cas de la matérialisation de photons d'énergie supérieure à $50 \mathrm{MeV}$, seule une détection séparée des électrons de matérialisation et de l'électron de recul permet d'obtenir une bonne précision sur tous les paramètres. En effet, à ces énergies, les électrons de la paire sont émis vers l'avant avec une énergie de quelques dizaines de $\mathrm{MeV}$ alors que l'électron de recul, d'énergie inférieure à $1 \mathrm{MeV}$ dans $90 \%$ des cas, est émis avec un angle $\theta_{\boldsymbol{r}}$ pouvant atteindre $90^{\circ}$.

Ces différentes raisons nous ont amenés à réaliser auprès de l'Accélérateur Linéaire d'Orsay une étude complète de la production de triplets. Nous allons décrire le dispositif expérimental utilisé. Il comporte d'une part une chambre à streamers remplie d'hélium gazeux (qui joue le rôle de cible), d'autre part, un spectromètre de paires constitué d'un aimant d'analyse et de quatre chambres à étincelles. La chambre à streamers permet d'obtenir les informations $q$ et $\theta_{\text {r }}$ sur l'électron de recul, le spectromètre de paires fournit les caractéristiques de la paire : les énergies $E^{+} \mathrm{du}$ positon, $E^{-}$du négaton, $k$ du photon $\left(k \simeq E^{+}+E^{-}\right)$ et l'angle d'ouverture de la paire [7].

Dans cet article, nous décrivons le spectromètre de paires en insistant plus particulièrement sur le système de lecture des chambres à étincelles et sur la séquence d'acquisition des données. Nous vérifions enfin le bon fonctionnement du dispositif et discutons de la précision obtenue sur l'énergie et l'ouverture angulaire.

2. Principe. - Un faisceau de photons obtenus par rayonnement de freinage d'électrons de $250 \mathrm{MeV} / \mathrm{c}$ est filtré par une colonne d'hydrure de lithium qui élimine la composante basse énergie du spectre. Le diamètre du faisceau est ramené à $8 \mathrm{~mm}$ par une série de collimateurs. Un aimant balai placé en avant du dispositif expérimental proprement dit (Fig. 1), permet d'éliminer les particules chargées produites dans les collimateurs.

Une chambre à -streamers de petites dimensions $\left(340 \times 110 \times 76 \mathrm{~mm}^{3}\right)$ est placée entre deux bobines situées en position de Helmholtz; dans le champ magnétique créé $(0,08 \mathrm{~T})$ l'électron de recul suit une trajectoire hélicoïdale qui est photographiée. Un autre avantage de ce champ magnétique coaxial au faisceau de photons est d'avoir une influence négligeable sur les électrons de matérialisation; en effet, ce champ est 
très faible et les électrons de la paire sont très peu inclinés sur son axe. Le spectromètre de paires comportant un électro-aimant et quatre chambres à étincelles (Fig. 2) en ligne avec un calculateur Varian 620 i permet l'étude des deux électrons de matérialisation. La chambre à streamers et les chambres à étincelles sont déclenchées par coïncidence sur les électrons de la paire. Un système de marquage permet d'associer chaque photo aux données correspondantes des chambres à étincelles [7].

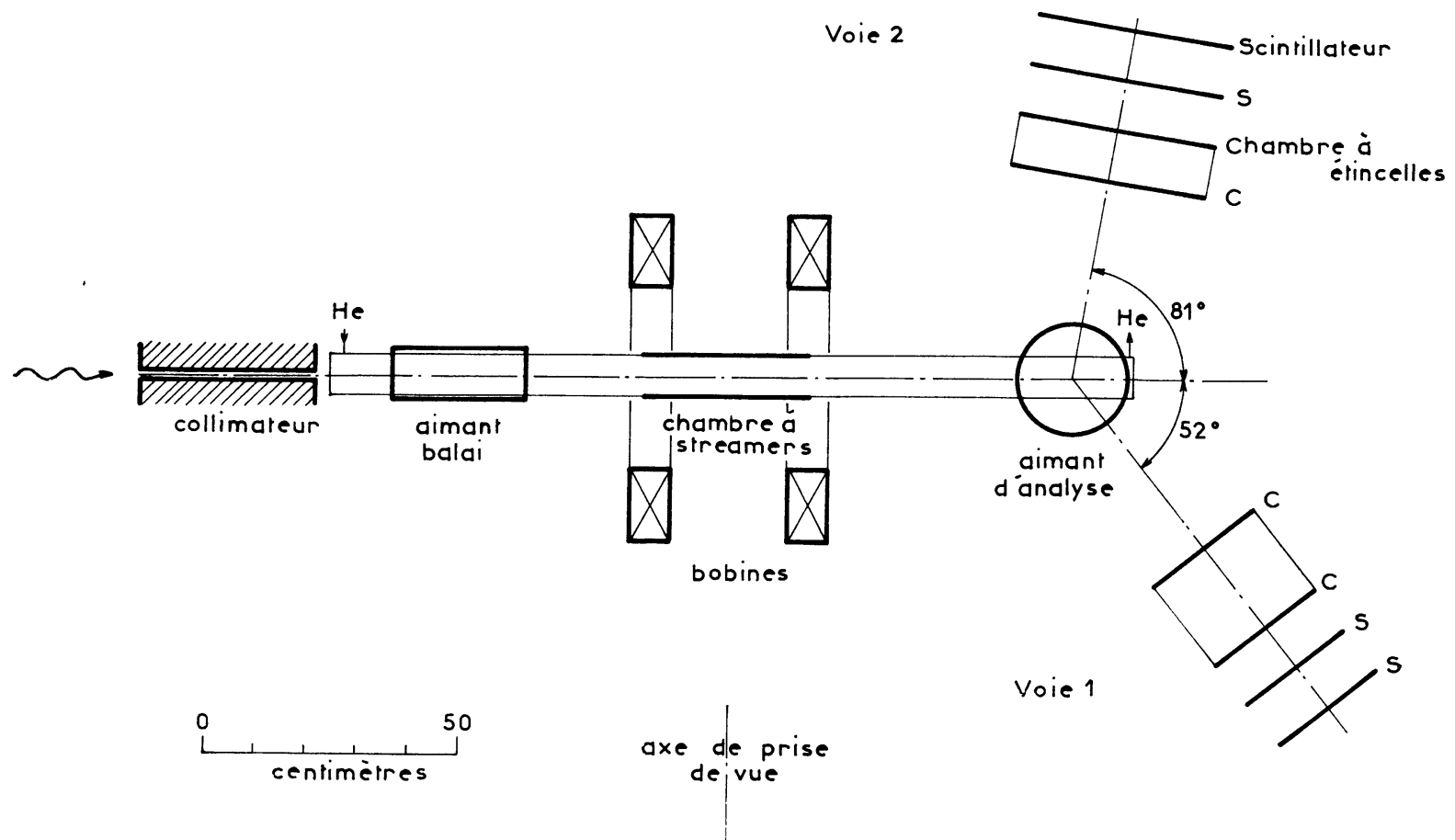

Fig. 1. - Schéma du dispositif expérimental.

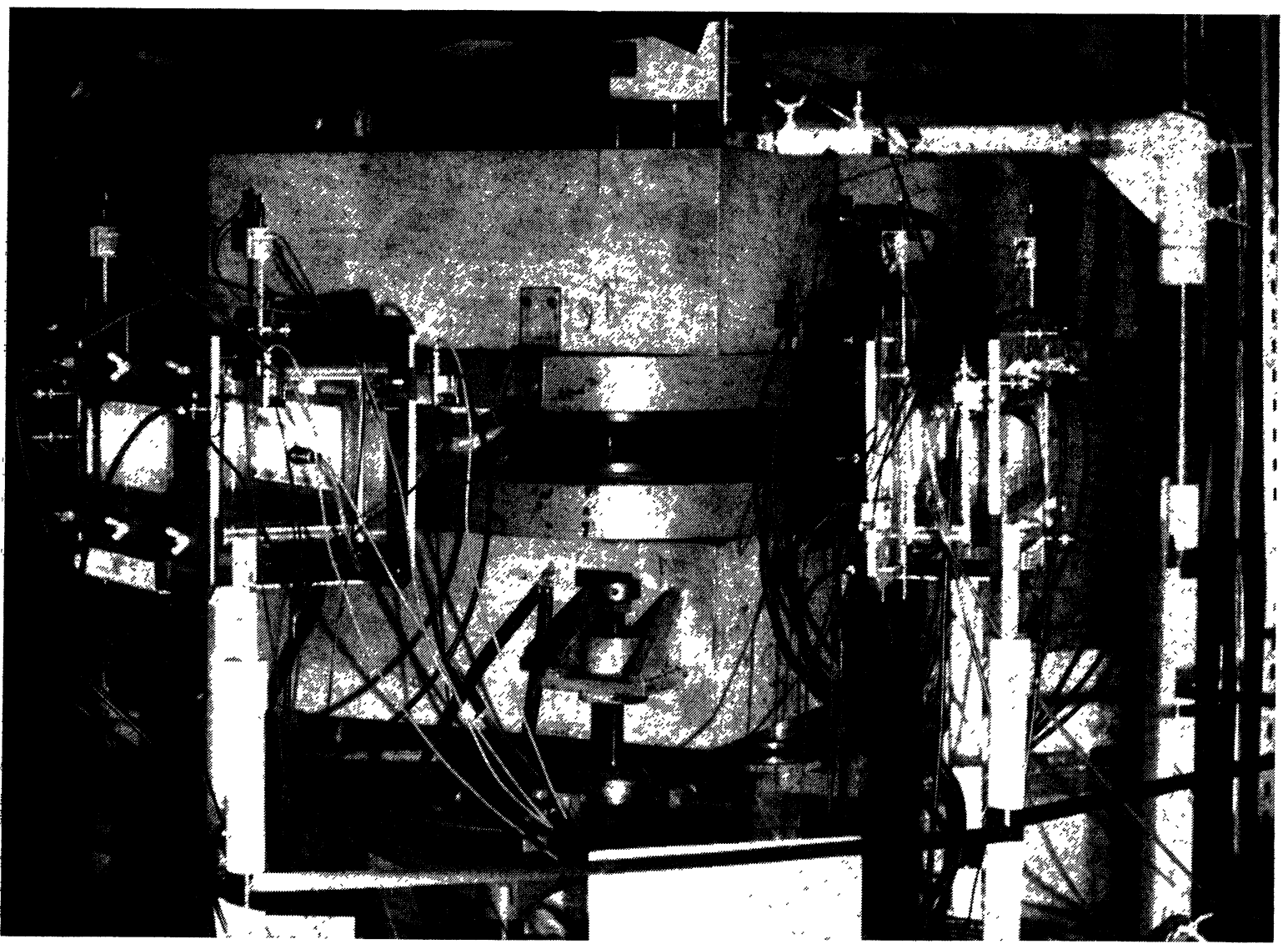

FIG. 2. - Photographie du spectromètre. 
3. Spectromètre. - L'analyse en énergie des électrons de matérialisation est effectuée au moyen d'un électro-aimant en $\mathrm{C}$ à champ homogène, à pôles circulaires de diamètre $210 \mathrm{~mm}$ et d'entrefer $40 \mathrm{~mm}$. Les électrons de matérialisation prenant naissance dans la chambre à streamers sont déviés par le champ magnétique et détectés par quatre scintillateurs " SPF » de $5 \mathrm{~mm}$ d'épaisseur. Ces scintillateurs (deux par voie) sont couplés à des photomultiplicateurs montés en coïncidence, permettant ainsi le déclenchement de quatre chambres à étincelles à fils à lecture magnétostrictive (2 par voie). Les deux coordonnées sont obtenues simultanément par lecture des deux plans de fils (HT et masse) de chaque chambre Afin de simplifier les problèmes d'alignement les deux chambres équipant chaque voie sont solidaires mécaniquement et forment une enceinte gazeuse unique [8]. Leurs dimensions sont : Voie gauche : 2 chambres identiques de $380 \times 90 \mathrm{~mm}^{2}$ distantes de $100 \mathrm{~mm}$; Voie droite : 2 chambres identiques de $240 \times 90 \mathrm{~mm}^{2}$ distantes de $200 \mathrm{~mm}$; distance entre 2 plans de fils : $8 \mathrm{~mm}$; distance entre 2 fils : $1 \mathrm{~mm}$.

L'appareillage détecte, pour un champ de 1,2 $\mathrm{T}$, les électrons d'énergie comprise entre 50 et $100 \mathrm{MeV}$ pour une voie, et entre 100 et $160 \mathrm{MeV}$ pour l'autre. Cette asymétrie permet ainsi par inversion du champ magnétique la détection des électrons et positons entre 50 et $160 \mathrm{MeV}$.

L'ensemble des informations (photographie de la chambre à streamers et données des chambres à étincelles), permet de reconstituer les trajectoires des particules dans l'espace et de remonter ainsi à leur énergie et à l'angle d'ouverture de la paire.

Dans ces conditions, et étant donné le domaine d'énergie de photons utilisé (150-260 MeV) pour lequel l'angle d'ouverture le plus probable est de l'ordre du degré, le spectromètre accepte pratiquement toutes les paires ayant une divergence énergétique comprise entre 0,3 et 0,7 . Les seules paires rejetées par le dispositif sont celles qui, émises dans un plan voisin du plan vertical, ont un angle d'ouverture supérieur à $3^{\circ}$.

4. Electronique de déclenchement. - Le schéma de principe de l'électronique associée au dispositif est représenté figure 3 .

Le faible temps de mémoire des chambres à étincelles (environ $1 \mu \mathrm{s}$ ) et la longueur des liaisons entre la salle de cible et la salle de contrôle $(60 \mathrm{~m})$ nous obligent à utiliser 2 chaînes distinctes : la première placée dans la salle de cible, à proximité immédiate du dispositif expérimental analyse les impulsions issues des photomultiplicateurs grâce à un ensemble de circuits logiques rapides qui déclenchent, dans le cas d'une coïncidence quadruple, la chambre à streamers, les chambres à étincelles et le dispositif d'acquisition des données. Certaines de ces impulsions sont transmises pour comptage vers la salle de contrôle où une deuxième voie d'analyse indépendante permet à l'opérateur de contrôler le bon fonctionnement de la voie principale.

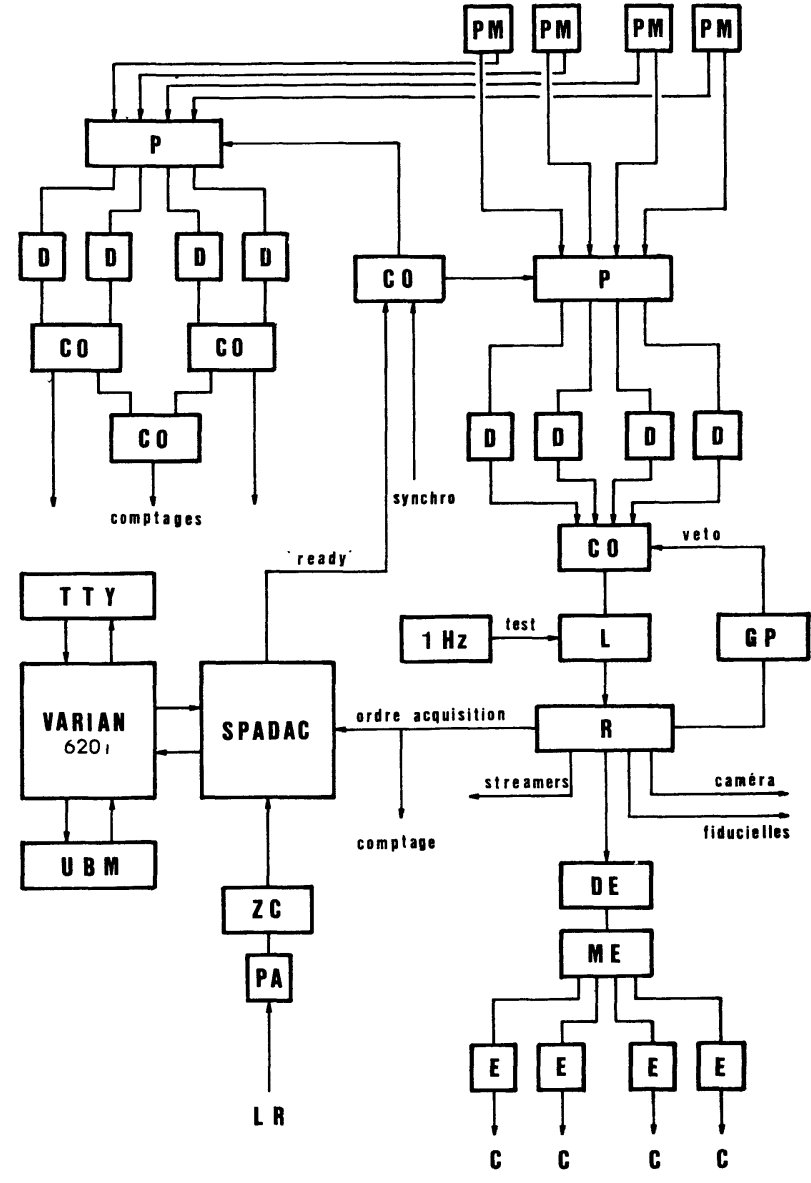

FIG. 3. - Schéma de principe de l'électronique.

P : Porte linéaire.

D : Discriminateur.

CO : Coïncidence.

L : Mélangeur.

R : Répartiteur.

$\mathrm{DE}$ : Déclencheur.

ME : Maître-éclateur.

GP : Générateur de porte.

E : Eclateur.

C : Chambre à étincelle.

PA : Préamplificateur.

ZC : Zéro crosser.

TTY : Télétype.

UBM : Unité de bandes magnétiques.

Le déclencheur [9] est un générateur de Marx qui utilise six transistors RT 1116 fonctionnant en régime d'avalanche; l'impulsion obtenue en sortie a une amplitude de $1000 \mathrm{~V}$ et un temps de montée de $5 \mathrm{~ns}$; elle attaque la grille d'un tube à cathode froide (EGG KN 22) qui pilote un maître éclateur (EGG GP 17b). Celui-ci dirige quatre éclateurs secondaires associés aux quatre chambres à étincelles permettant ainsi un réglage indépendant pour chaque chambre (Fig. 4).

5. Lecture des chambres à étincelles. - Chaque plan de fils est lu au moyen d'une ligne à retard magnétostrictive ; le préamplificateur de lecture (Fig. 5) solidaire d'une barrette de laiton contenant le fil magnétostrictif, se compose essentiellement d'un 


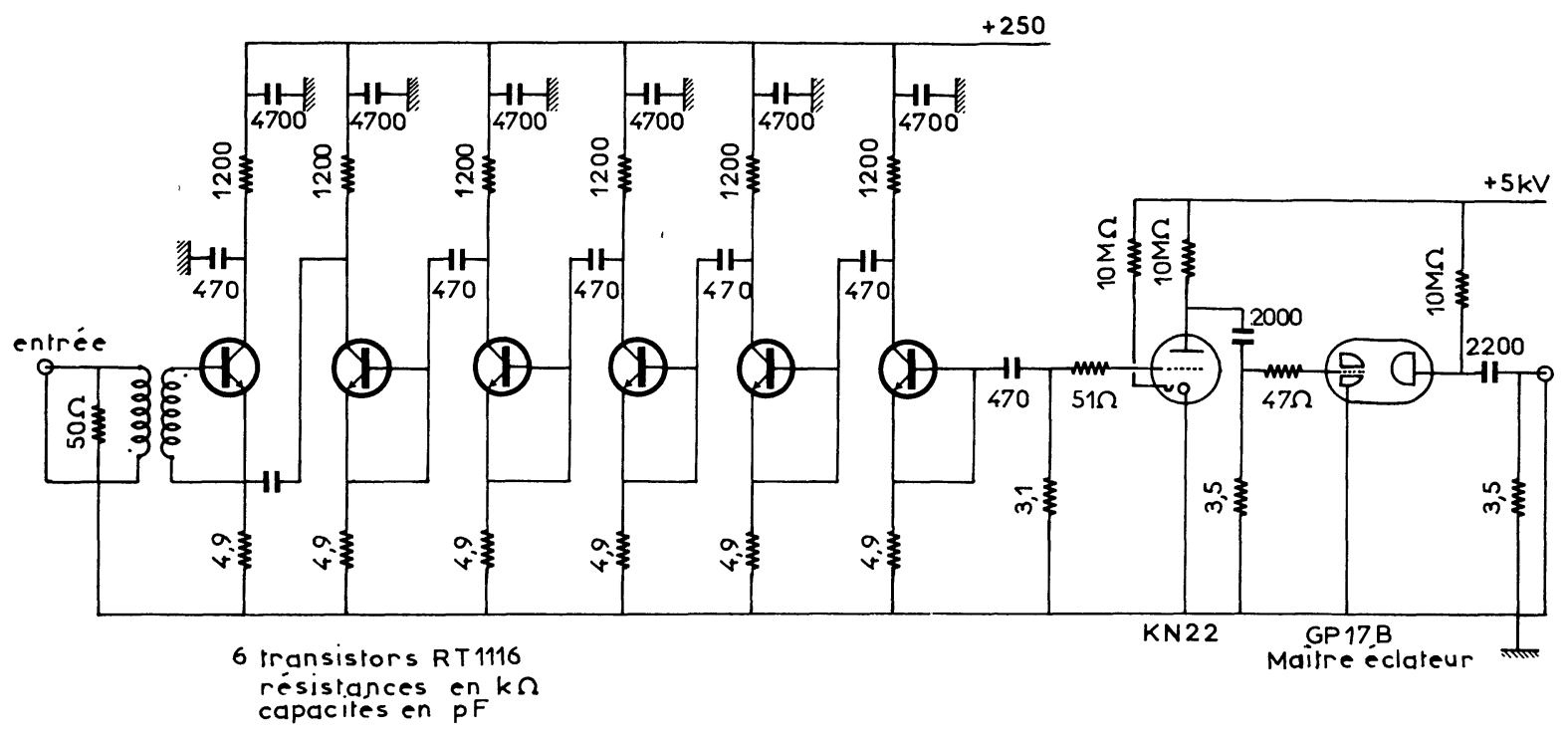

FIG. 4. - Chaîne de déclenchement.

amplificateur différentiel $\mu \mathrm{A} 702$ suivi d'un transistor dont la polarisation permet d'éliminer les impulsions dues aux réflexions mécaniques sur les extrémités de la barrette. En effet, ces impulsions ont des amplitudes beaucoup plus faibles que celles des signaux dus aux ondes directes. Le gain du préamplificateur est réglé en agissant sur la valeur des tensions d'alimentation. Le signal de sortie est négatif, son amplitude est de $2 \mathrm{~V}$ et sa durée est de $400 \mathrm{~ns}$. Afin d'augmenter la précision de la localisation, ce signal est dérivé et un dispositif de passage à zéro [10] délivre une impulsion standard négative d'amplitude $1,2 \mathrm{~V}$ et de durée $100 \mathrm{~ns}$. $\mathrm{Ce}$ signal est transmis jusqu'à la salle de contrôle où se trouve le dispositif d'acquisition des données.

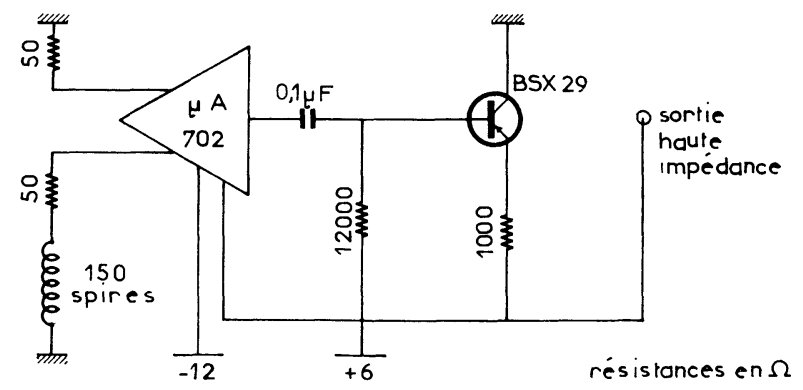

Fig. 5. - Préamplificateur de lecture.

Aux extrémités de chaque barrette, deux fils de référence dont la distance peut être repérée optiquement permettent l'étalonnage de la ligne à retard. A chaque déclenchement apparaissent, à la sortie de chaque ligne à retard, trois signaux correspondant respectivement au premier fil de référence, à l'étincelle et au second fil de référence. Le pouvoir de résolution d'une ligne à retard et des circuits électroniques associés est de $0,5 \mathrm{~mm}$ dans le cas d'une étincelle unique.
6. Acquisition des données. - La séquence d'acquisition des données s'effectue de la façon suivante (Fig. 6) :

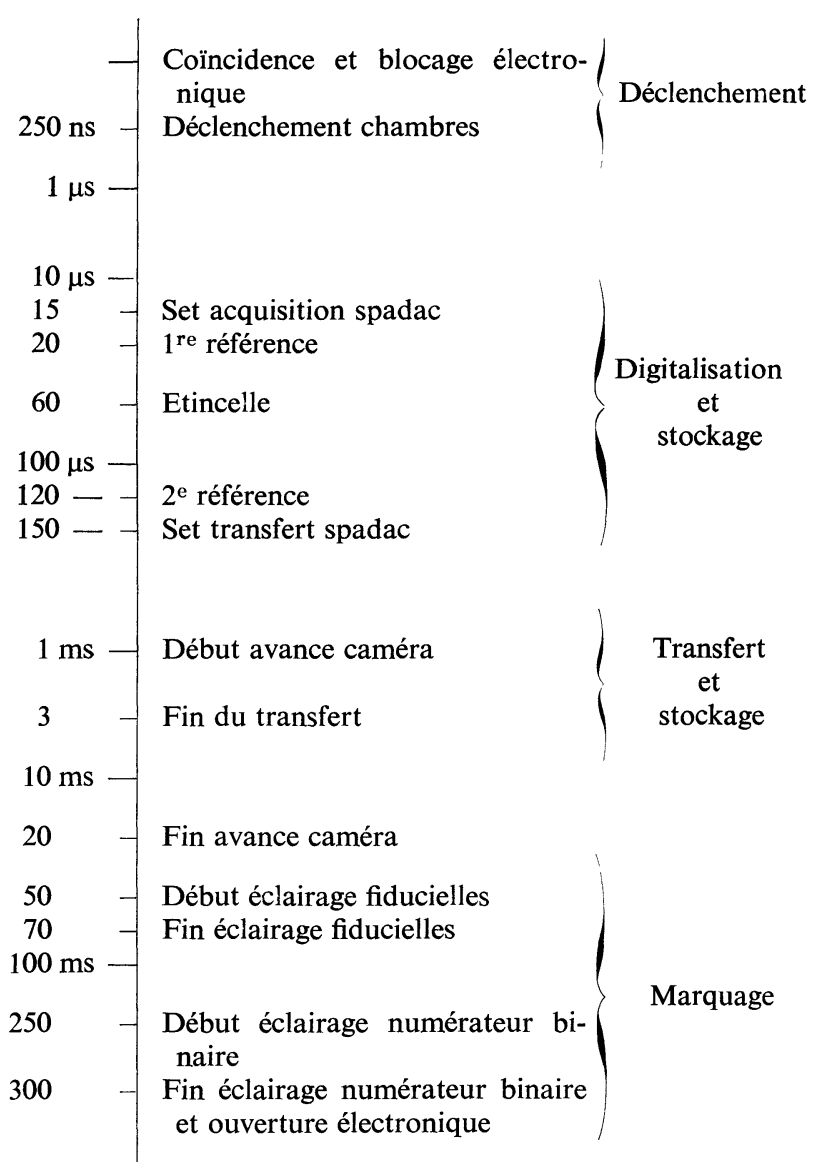

Fig. 6. - Séquence d'acquisition des données.

6.1 DéClenChement de la chambre à streamers et des chambres à étincelles. 
6.2 Digitalisation de la position des étincelles au moyen du système d'acquisition de données Spadac [11]. Deux modules de digitalisation sont affectés à la lecture des données d'un plan de fils ; l'impulsion de déclenchement des chambres à étincelles commande le départ d'une horloge $20 \mathrm{MHz}$ et le signal dû à la première référence ouvre la porte des 2 modules qui commencent à compter les impulsions d'horloge; le premier est stoppé par l'arrivée du signal d'étincelle tandis que le second compte jusqu'à l'apparition du signal dû au deuxième fil de référence. Nous avons alors dans les compteurs deux nombres proportionnels respectivement à la distance séparant la première référence de l'étincelle et à la distance entre les deux références. Par ailleurs, des échelles de comptage simples permettent d'enregistrer les impulsions issues de l'électronique rapide (comptage des impulsions de chaque photomultiplicateur, nombre de coïncidences, nombre de cycles de l'accélérateur, etc...).

6.3 Transfert des données contenues dans les échelles du Spadac vers le calculateur Varian $620 \mathrm{i}$. Celui-ci a deux fonctions principales [12] :

- D'une part, il accumule les informations dans une mémoire tampon et les enregistre sur bandes magnétiques, par paquets de 15 événements (420 mots). Les informations comprennent :

a) Les données relatives aux matérialisations (les coordonnées des points de passage des particules dans les quatre chambres à étincelles, le nombre de coïncidences, le nombre de coups sur chacun des quatre photomultiplicateurs).

b) Le contenu d'un compteur servant à numéroter les événements qui ont été enregistrés depuis le début de l'expérience.

c) Le contenu d'un numérateur binaire à quatre lampes qui est photographié en même temps que la chambre à streamers. (Ce numérateur permet d'associer chaque photographie d'événement avec chaque déclenchement des chambres à étincelles, son affichage est commandé directement par l'ordinateur.).

d) Le numéro du film en cours de prise de vue.

- D'autre part, il permet à l'expérimentateur de vérifier le bon fonctionnement du dispositif de détection et d'effectuer le cas échéant les réglages électroniques. Il est par exemple possible de faire imprimer sur un télétype au fur et à mesure de leur arrivée les quatre données $a), b), c), d$ ), ci-dessus ou de passer en fonctionnement « test "; à chaque événement, les données sont transcrites par le télétype mais ne sont pas gardées en mémoire. Ce mode de fonctionnement donne la possibilité d'effectuer un réglage en utilisant un générateur $1 \mathrm{~Hz}$ pour déclencher les chambres (Fig. 3). De plus pour s'assurer que les valeurs des références n'évoluent pas dans le temps, le programme nous fournit les spectres des huit références associées aux quatre chambres à étincelles. Le programme signale aussi la fin de chaque film.

A la fin de chaque séquence, le film est avancé et le numérateur à quatre positions binaires est incrémenté.

7. Conclusion. - Le dispositif a permis l'acquisition de 35000 événements de matérialisation dans la partie située entre l'aimant balai et l'aimant d'analyse ( $\simeq 1 \mathrm{~m}$, Fig. 1). Parmi ceux-ci, 8000 paires ou triplets prennent naissance dans la partie de la chambre à streamers visible par la caméra $(\sim 0,25 \mathrm{~m})$. La connaissance du point de matérialisation et des trajectoires de particules dans le spectromètre permet de déterminer l'énergie de chacune des branches de la paire $\left(E^{+}, E^{-}\right)$ l'énergie du photon incident $\left(k \simeq E^{+}+E^{-}\right)$, les angles $\left(\omega_{1}, \omega_{2}\right)$ d'émission des électrons par rapport au faisceau de photons incidents et l'ouverture angulaire de la paire $(\omega)$.

Un dépouillement partiel nous a permis de vérifier le bon fonctionnement du spectromètre. Etant donné le domaine d'énergie des photons utilisés $(\simeq 150$ à $260 \mathrm{MeV}$ ), l'ouverture angulaire des paires de matérialisation est de l'ordre du degré ; par conséquent, les distances $d_{1}$ et $d_{2}$ du centre de l'aimant au prolongement des trajectoires électroniques déterminées par les chambres à étincelles (Fig. 7) doivent être faibles. La

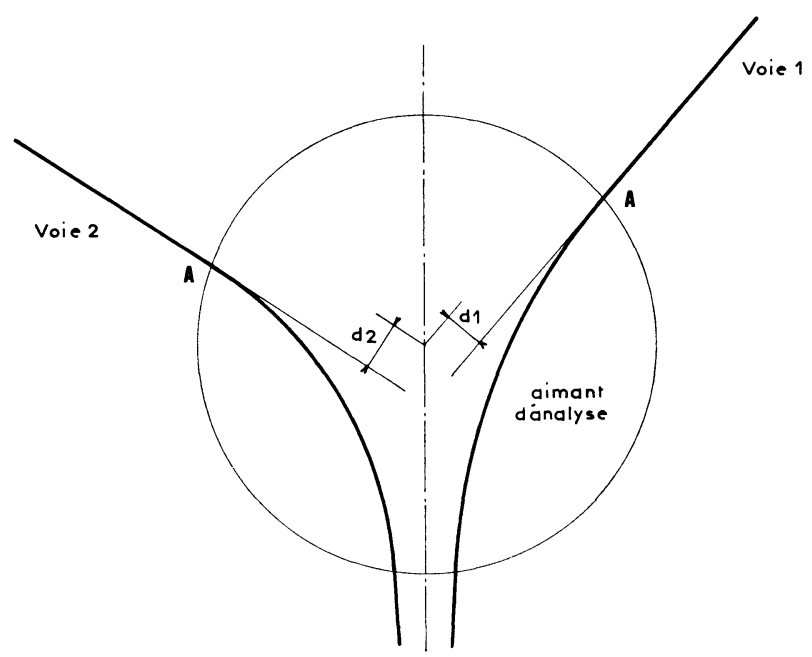

Fig. 7. - Distances du centre de l'aimant à la prolongation des trajectoires électroniques dans les chambres.

figure 8 représente les histogrammes de ces distances sur les deux voies. Nous remarquons que 77 et $57 \%$ des événements donnent une distance inférieure à $1 \mathrm{~cm}$. La différence entre les voies s'explique facilement en comparant les distributions des angles $\omega_{1}$ et $\omega_{2}$ (en effet, si $\omega_{p}$ désigne la valeur de l'angle le plus probable, nous avons $\omega_{1 p}<\omega_{2 p}$ ).

Les distributions du point de passage (A) des particules suivant l'axe du champ magnétique à la sortie de l'aimant équivalent sont représentées figure 9. Elles sont symétriques par rapport à l'axe du faisceau et $90 \%$ des points sont situés dans un intervalle $(-2 \mathrm{~cm}$, $+2 \mathrm{~cm}$ ) correspondant à l'entrefer de l'aimant d'analyse. 

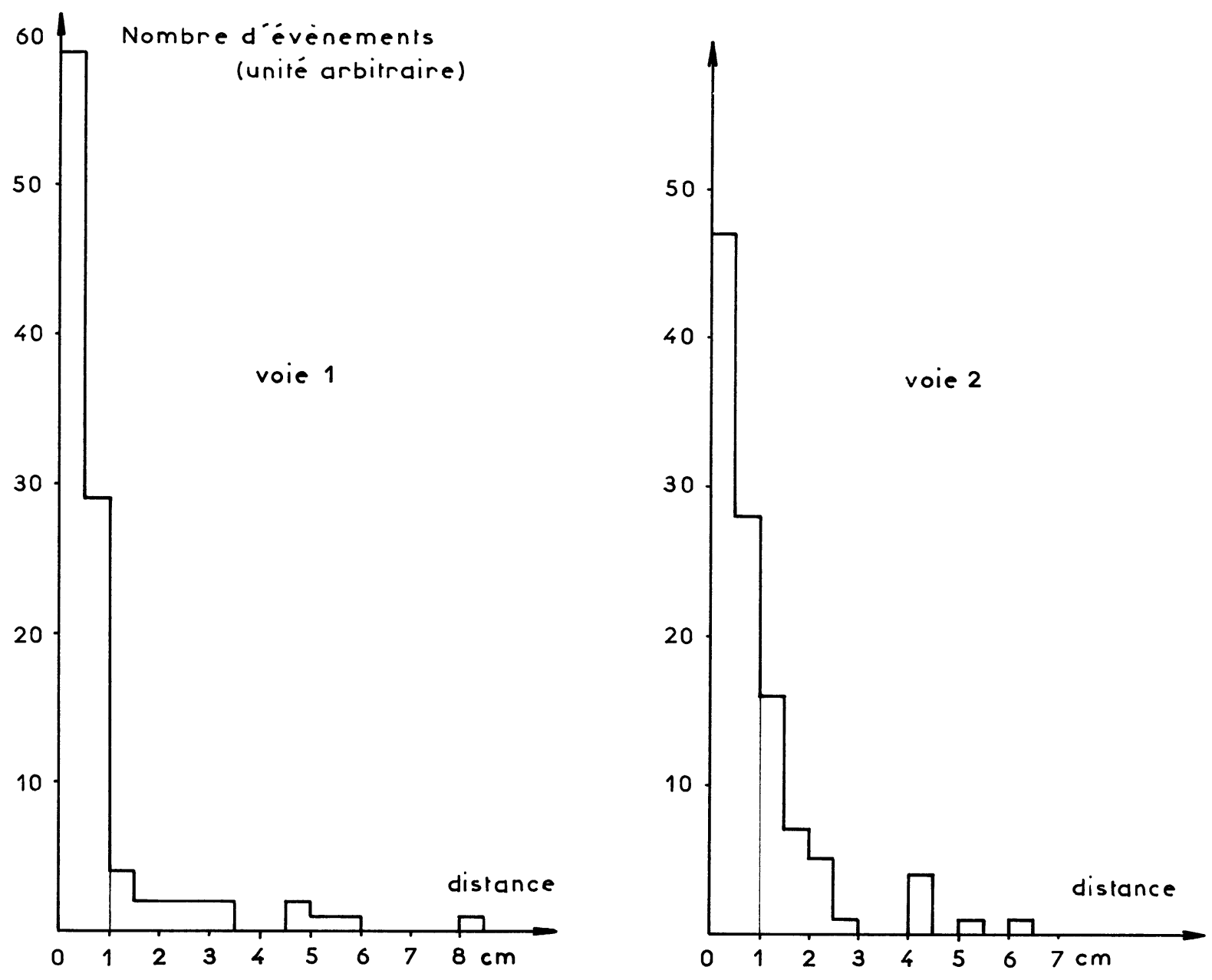

FIG. 8. - Histogrammes des distances du centre de l'aimant à la prolongation des trajectoires dans les chambres.
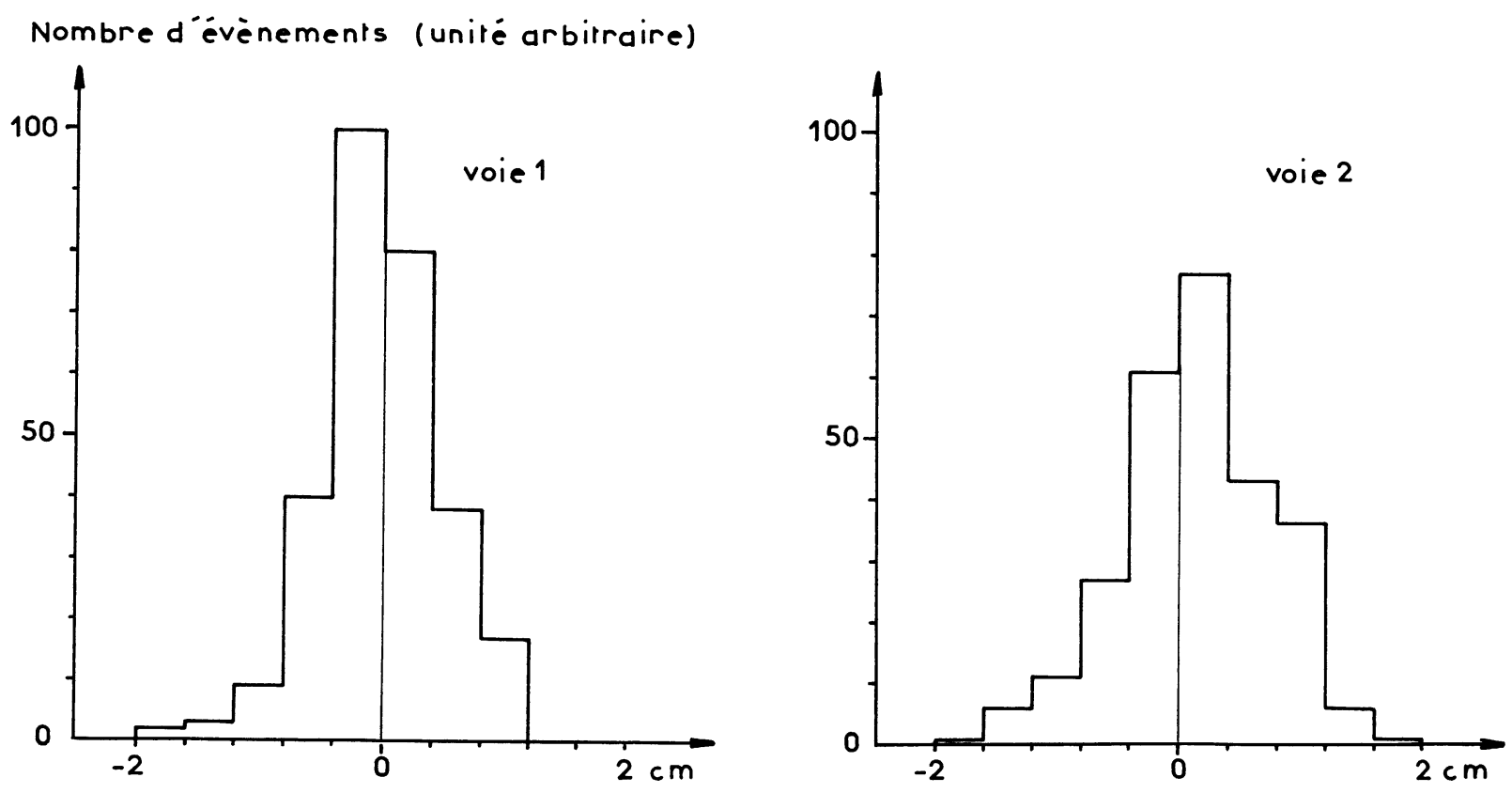

Fig. 9. - Histogramme de la côte du point $\mathrm{A}$ de passage des particules à la sortie de l'aimant équivalent. 
Afin de vérifier la validité des résultats obtenus à l'aide d'un programme permettant de déterminer l'énergie des électrons et l'angle d'ouverture de la paire à partir des données des chambres, nous avons reconstruit géométriquement 50 événements de matérialisation. Nous constatons que toutes les trajectoires obtenues sont correctes.

Dans cette expérience, la précision sur l'énergie des électrons de la paire dépend de plusieurs facteurs, à savoir :

- La position de l'étincelle déterminée avec une erreur inférieure au millimètre si les trajectoires des particules ont une inclinaison inférieure à $15^{\circ}$ par rapport à la normale des chambres à étincelles. Cette condition est réalisée dans $92 \%$ des cas sur la voie la plus énergétique et dans $70 \%$ des cas sur l'autre. L'erreur due à cette imprécision est toujours inférieure à $1 \%$.

- L'erreur commise dans la mesure des distances entre le centre de l'aimant et les chambres à étincelles introduit une imprécision inférieure à $0,5 \%$. L'imprécision dans la détermination de la position de l'origine de matérialisation par rapport à l'aimant introduit une erreur négligeable sur la valeur de l'énergie.

L'inhomogénéité du champ magnétique analyseur introduit une erreur moyenne de l'ordre de $2 \%$ sur la valeur de l'énergie.

On constate donc que l'ensemble des erreurs concernant la mesure de l'énergie reste inférieure à $4 \%$.

Le maximum de la distribution de l'ouverture angulaire $\omega$ des paires est situé dans la bande $0,5-1^{\circ}$. Cette valeur correspond à celle obtenue par la formule $\omega_{p} \simeq 4 m_{0} c^{2} / k\left(m_{0} c^{2}\right.$ étant l'énergie de l'électron au repos). Parmi les différentes incertitudes dans la détermination de ces angles, la principale évaluée à environ
$15 \%$ est due à l'imprécision sur la position de l'étincelle dans la chambre. A notre connaissance, pour le domaine d'énergie de photons étudié, seule l'expérience de Hart et al. [4] permet d'obtenir l'ouverture angulaire projetée avec une erreur d'environ $1 / 4^{\circ}$ ce qui correspond à une incertitude de $25 \%$ pour un angle de $1^{\circ}$.

Il faut remarquer que l'on aurait pu réduire l'erreur sur l'ouverture angulaire en augmentant la distance entre les deux chambres dans chaque voie d'analyse. Ceci aurait pour conséquence de réduire les bandes d'énergie acceptées par le dispositif.

Nous avons gardé un domaine d'énergie relativement étendu car la comparaison, plutôt que l'étude directe, des ouvertures angulaires de paires et de triplets, était l'un des buts recherchés. Comme il n'existe pas de distribution théorique de l'ouverture angulaire de triplet, cette comparaison est importante.

Ainsi, ce dispositif permet à l'aide des données fournies par le spectromètre, non seulement de déterminer le rapport de la section efficace de triplets à celle de paires pour différentes énergies de photons [13], de comparer l'ouverture angulaire des paires à celle des triplets, mais encore de déterminer à l'aide de la chambre à streamers l'énergie et l'angle d'émission du recul.

Remerciements. - Nous tenons à remercier M. le Professeur A. Lagarrigue d'avoir mis à notre disposition les équipements du Laboratoire de l'Accélérateur Linéaire, MM. B. Milman, pour l'attention avec laquelle il a suivi l'expérience, B. Grossetête, pour l'aide qu'il nous a apportée lors de l'implantation, G. Danon et A. Damany, qui nous ont fourni le schéma du déclencheur, ainsi que tout le personnel de l'Accélérateur.

\section{Bibliographie}

[1] Mork K. J., Phys. Rev. 160 (1967) 1065.

[2] Castor J., Thèse $3^{\mathrm{e}}$ cycle $\mathrm{n}^{\mathrm{o}} 162$, Faculté des Sciences, Clermont-Ferrand (1969).

[3] Borsellino A., Rev. Univ. Nacl. Tucuman A 6 (1947) 7.

[4] Hart E. L. et al., Phys. Rev. 115 (1959) 678.

[5] Gates D. C. et al., Phys. Rev. 125 (1962) 1310.

[6] Benaksas D., Morrison R., Phys. Rev. 160 (1967) 1245.

[7] Castor J., Devaux A., Lieutier M., Marand F., Rapport Interne, Physique Nucléaire, ClermontFerrand, mars 1969.

Castor J., Devaux A., Lieutier M., Marand F. et Bertin P. Y., Rapport Interne, Physique Nucléaire, Clermont-Ferrand, avril 1969.
Castor J., Bertin P. Y., Devaux A., Lieutier M., ISABELle D. B., Proposition d'expérience présentée à l'Accélérateur Linéaire d'Orsay, juillet 1969.

[8] Planchard E. J., Thèse de $3^{\mathrm{e}}$ cycle, $\mathrm{n}^{\circ}$ 256, Faculté des Sciences, Clermont-Ferrand (1971).

[9] D'après service d'instrumentation électronique, Laboratoire de l'Accélérateur Linéaire d'Orsay (91).

[10] D'après ChIKovaNi et al., Rapport CERN 67-13.

[11] SPADAC, SEN Electronique, Genève, Suisse.

[12] Devaux A., Thèse de $3^{\mathrm{e}}$ cycle, $\mathrm{n}^{\circ}$ 257, Faculté des Sciences, Clermont-Ferrand (1971).

[13] Castor J., Devaux A., Fleury A., Lieutier M., Planchard E., Bertin P. Y., Isabelle D. B. J. Physique 32 (1971) C5b-267. 